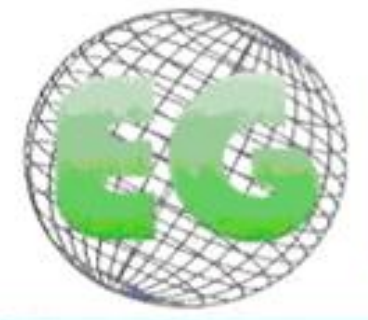

No 39

www.um.es/egloball

\title{
CLÍNICA
}

\section{Efectividad de la visita prequirúrgica sobre la ansiedad, el dolor y el bienestar}

Effectiveness of preoperative visit on anxiety, pain and wellbeing

\section{*Bagés Fortacín, Cristina **Lleixà Fortuño, María del Mar ${ }^{* * *}$ Español Puig, Cristina ${ }^{* * *}$ Imbernón Casas, Gloria ${ }^{* * *}$ Munté Prunera, Nuria ${ }^{* * *}$ Vázquez Morillo, Dolores}

\begin{abstract}
*Enfermera Supervisora del Área Quirúrgica del Hospital Sant Pau i Santa Tecla. E-mail: cbages@xarxatecla.cat **Profesora Departamento de Enfermería, Universidad Rovira i Virgili Campus Terres de l'Ebre ${ }^{* * *}$ Enfermera instrumentista del Área Quirúrgica del Hospital Sant Pau i Santa Tecla ****Enfermera asistencial del Área Quirúrgica del Hospital Sant Pau i Santa Tecla. Tarragona. España.
\end{abstract}

Palabras Clave: Visita prequirúrgica; ansiedad; cirugía programada; dolor; bienestar; Caring. . Keywords: Preoperative visi; anxiety; elective surgery; well-being; Caring

\section{RESUMEN}

Objetivo principal: Evaluar si la visita prequirúrgica es una herramienta eficaz para disminuir el nivel de ansiedad en el preoperatorio y el dolor postoperatorio.

Metodología: Ensayo controlado de dos grupos aleatorios de medidas repetidas antes-después. El estudio consiste en comparar las medias de los niveles de ansiedad, dolor y bienestar en diferentes momentos del proceso quirúrgico. Para el análisis bivariante se han utilizado el test no paramétrico de U de Mann Whitney y el test de Chi Cuadrado de Paerson. Para comprobar la asociación entre las variables cuantitativas se ha utilizado la correlación Rho de Spearman.

Resultados: El grupo intervención presenta un descenso estadísticamente significativo del nivel de ansiedad en el preoperatorio, del dolor postoperatorio y un aumento del bienestar.

Conclusión: La visita prequirúrgica es una herramienta eficaz para disminuir la ansiedad situacional y el dolor postoperatorio.

\section{ABSTRACT}

Objective: Assess whether the preoperative visit is an effective instrument to reduce both the preoperative anxiety level and postoperative pain. 
Methods: Randomized controlled trial with two groups, repeated measures before-after. The study consists of comparing the average levels of anxiety, pain and well-being at different times of the surgical process. The non-parametric tests of $U$ Mann-Whitney and Chi-Square Pearson were used for the bivariate analysis and Spearmans's Rho correlation was used to check the association between quantitative variables

Results: The experimental group shows a statistically significant decrease of the preoperative anxiety level and postoperative pain and an increase of wellbeing

Conclusions: The preoperative visit is an effective instrument to reduce the situational anxiety level and postoperative pain.

\section{INTRODUCCIÓN}

La persona que tiene que ser intervenida quirúrgicamente pasa a formar parte de un sistema totalmente desconocido que implica ciertos riesgos ${ }^{1,2}$. Esta vivencia puede ocasionarle en cierta medida una serie de reacciones y manifestaciones tales como inseguridad, miedo, nerviosismo y ansiedad ${ }^{2-5}$. Si la ansiedad y el estrés sobrepasan el límite deseado, se pueden desencadenar los aspectos negativos de la ansiedad ${ }^{6}$. Son muchos los estudios que afirman que cuanto más intensa es la ansiedad antes de una intervención quirúrgica, más larga y difícil puede ser la convalecencia, aumentando la percepción del dolor postoperatorio y, consecuentemente, condicionando el bienestar de las personas ${ }^{2,6-15}$.

Esta situación se agrava por el hecho de estar inmerso en un entorno que puede considerarse hostil, debido a la estructura y organización del quirófano: un ambiente frío, aséptico y altamente tecnificado, rodeado de alarmas, aparatos sofisticados y luces potentes ${ }^{16}$, coyuntura que se intensifica todavía más si el paciente es operado con anestesia loco-regional ${ }^{17-19}$. Todas estas circunstancias que rodean la intervención quirúrgica pueden ser percibidas por el paciente como una amenaza y producirle un sentimiento de ansiedad.

El acto quirúrgico no afecta sólo a nivel físico y psicológico, sino que también afecta a nivel social y espiritual si lo analizamos desde una perspectiva holística ${ }^{7,16,20-23}$. Un referente en el proceso del cuidado holístico y humano es Jean Watson y su teoría del Caring ${ }^{24-27}$. Watson afirma que "...tanto la formación en enfermería como el sistema de suministro de cuidados de salud deben basarse en los valores humanos y deben preocuparse por el bienestar de los demás..."28. La idea del Caring es aliviar el sufrimiento humano en una relación caracterizada por la responsabilidad y el deseo de hacer el bien, así como preservar la vida y la salud. En el entorno pre y perioperatorio el caring brinda la posibilidad de crear un vínculo entre el paciente y la enfermera. Desde la perspectiva del paciente, la enfermera es "alguien que me cuida, crea una atmósfera de calma y en quien puedo confiar"29.

Con la comunicación y el diálogo la enfermera estimula al paciente a verbalizar y exponer sus sentimientos y temores ${ }^{30}$, a la vez que muestra su disposición escuchando con atención y respeto. De esta manera puede identificar las necesidades de la persona y percibir su angustia, sus dudas y temores, proporcionando ayuda y soporte durante todo el proceso quirúrgico. Es en este momento cuando el rol de la enfermera es más importante al brindar una atención directa y de buena calidad. 
Para conseguir unos resultados positivos en el control de la ansiedad y mejorar el bienestar es necesario dar toda la información posible, y una medida efectiva es establecer la visita prequirúrgica dentro del proceso preoperatorio.

Se entiende como visita prequirúrgica el proceso de personalización de cuidados entre la enfermera de quirófano y el paciente antes de una intervención programada, mediante una entrevista personalizada, con el objetivo de proporcionar información, conocimientos de autocuidado, asesoramiento y disminuir la ansiedad y el temor ${ }^{1}$. Hay que tener en cuenta que una intervención quirúrgica afecta tanto al paciente como a toda la unidad familiar ${ }^{30}$, por lo que también hay que tener en consideración a la familia en el momento de realizar la visita prequirúrgica.

En la bibliografía sobre este tema hay controversia respecto a la eficacia de la visita prequirúrgica sobre la disminución del nivel de ansiedad. Ya en los años 60, un estudio de Egbert et al. ${ }^{31}$ evidenció que la información para disminuir la ansiedad preoperatoria era mucho más efectiva que la medicación con sedación. En el transcurso de los años, más estudios han corroborado esta afirmación, pero en la última década es cuando más se ha investigado sobre este tema ${ }^{6,7-9,19,32-43}$.

Los trabajos de Chiriviches et al. ${ }^{7}$, Aparcero et al. ${ }^{4}$ y Orihuela-Pérez et al. ${ }^{1}$ confirman que los pacientes que han recibido información adicional sobre el proceso quirúrgico mediante una visita prequirúrgica estructurada presentan niveles de ansiedad estacional inferiores estadísticamente significativos respecto a los que han recibido la información habitual. Por otro lado, los estudios de Díez-Álvarez et al. ${ }^{33}$, Marín Romero et al. ${ }^{20}$ y Navas ${ }^{44}$ afirman que la disminución de ansiedad obtenida después de recibir información adicional no es estadísticamente significativa.

Otros trabajos publicados hacen referencia al momento en que el paciente comienza a experimentar un aumento de la ansiedad. Hay estudios que afirman que la ansiedad se presenta como mínimo una semana antes del acto quirúrgico, y que se intensifica en las horas previas del ingreso, por lo que sería mucho mejor dar la información preoperatoria antes de que los pacientes ingresasen en el hospital ${ }^{2,6,19,34,44,45}$.

Incluso algunos autores, como Herrera-Espiñeira et al. ${ }^{46}$ y $\mathrm{Kindler}^{47}$, afirman que el nivel cultural y la edad están asociados a una peor comprensión de la información.

A pesar de la controversia de algunas investigaciones respecto la efectividad de la visita prequirúrgica sobre la ansiedad y el dolor, muchos trabajos coinciden en afirmar que la información y la educación que se suministra durante la visita previa a la intervención quirúrgica mejora la percepción de la satisfacción y del bienestar ${ }^{1,4,7,10,37,38,43,48-50}$.

\section{OBJETIVOS}

El objetivo de este trabajo fue analizar el efecto de la visita prequirúrgica estructurada de enfermería sobre el nivel de ansiedad, la evolución de la percepción del dolor postoperatorio y del bienestar en el proceso quirúrgico. De forma adicional se comprobó si existía asociación entre el nivel de ansiedad y el dolor, así como con la experiencia vivida en procesos quirúrgicos anteriores y la adecuación de la información recibida. 


\section{MATERIAL Y MÉTODO}

Ensayo clínico controlado, de medidas repetidas antes-después, de dos grupos aleatorios.

La población muestra estuvo formada por 30 pacientes en cada grupo, que ingresaron de forma programada en la unidad de hospitalización del Servicio de Cirugía Ortopédica y Traumatológica del Hospital Sant Pau i Santa Tecla de Tarragona, para ser intervenidos de Prótesis Total de Rodilla (PTR), Prótesis Total de Cadera (PTC) y Artrodesis Lumbar (AL). Se excluyeron los menores de 18 años, pacientes con deterioro cognitivo, pacientes psiquiátricos y extranjeros que no comprendieran ninguna de las dos lenguas oficiales de Catalunya (catalán y castellano), y pacientes oncológicos (ya que la ansiedad es inherente en este proceso, por lo que los niveles de ansiedad del estudio podrían resultar alterados). Los pacientes se reclutaron de forma consecutiva de enero a marzo de 2012.

El Comité Ético de Investigación Clínica del Hospital Universitario Joan XXIII de Tarragona aprobó el protocolo del estudio el 4 de Enero de 2012 (ref. CEIC 61/2011). Asimismo se obtuvo el permiso del Comité de Asesoramiento Científico del Hospital Sant Pau i Santa Tecla de Tarragona.

Como instrumentos de obtención de datos se utilizó el cuestionario de autoevaluación STAI Ansiedad Estado-Rasgo validado en versión española. La Ansiedad Estado $(\mathrm{A} / \mathrm{E})$ está conceptualizada como un estado o condición emocional transitoria que experimenta la persona en un momento determinado. La Ansiedad Rasgo (A/R) es un patrón de ansiedad que identifica un rasgo de personalidad ${ }^{51}$. La escala $A / E$ consta de 20 frases con las que el sujeto puede contestar cómo se siente en un momento particular y cuyas posibles respuestas son: nada 0 , algo 1, bastante 2, mucho 3; y la escala A/R consta a su vez de 20 frases con las que el sujeto puede mostrar cómo se siente generalmente, y las posibles respuestas son: casi nunca 0 , a veces 1 , a menudo 2 y casi siempre 3 . En ambos cuestionarios las frases se plantean con escala directa e indirecta que se han mezclado para evitar el efecto de aquiescencia en las respuestas. La puntuación total obtenida en cada cuestionario oscila entre 0 y 60 , que se transforma en centiles en función del sexo y la edad. Del mismo cuestionario A/E se destacó el ítem 20 para obtener la puntuación de la percepción del bienestar. Para medir el dolor se utilizó la Escala Visual Analógica (EVA). También se recogieron variables sociodemográficas como: edad, sexo, estado civil, convivencia, nivel de estudios e intervenciones quirúrgicas previas.

En el momento del ingreso, la tarde anterior a la intervención quirúrgica, la enfermera de la unidad de hospitalización daba la información estandarizada propia del servicio, tanto a los pacientes del grupo control como al grupo intervención. Posteriormente, una enfermera del área quirúrgica se desplazaba a la habitación de los pacientes de ambos grupos para informar por escrito del objetivo del estudio y solicitar la firma del consentimiento informado. De manera adicional, los pacientes del grupo intervención recibían la información específica de la visita prequirúrgica.

La recogida de datos se llevó a cabo en diferentes momentos del proceso: el día del ingreso, en la sala de preanestesia (PRE) y durante les primeras 72 horas postoperatorias (Tabla 1). 


\begin{tabular}{|c|c|c|c|c|c|}
\hline \multicolumn{3}{|c|}{ GRUPO CONTROL n=30 } & \multicolumn{3}{|c|}{ GRUPO INTERVENCIÓN n=30 } \\
\hline DÍA & LUGAR & DOCUMENTOS & DÍA & LUGAR & DOCUMENTOS \\
\hline $\begin{array}{l}\text { 1 }^{\text {er }} \text { día } \\
\text { Ingreso } \\
\text { 18:00 h. }\end{array}$ & $\begin{array}{l}\text { Habitación } \\
\text { planta }\end{array}$ & $\begin{array}{l}\text {-Información } \\
\text { estudio } \\
\text {-Firma } \\
\text { consentimiento } \\
\text {-Test STAI A/E + } \\
\text { A/R ingreso } \\
\text {-Datos socio- } \\
\text { demográficos }\end{array}$ & $\begin{array}{l}1^{\text {er }} \text { día } \\
\text { Ingreso 18:00 h. } \\
\text { INFORMACIÓN } \\
\text { VISITA } \\
\text { PREQUIRÚRGI } \\
\text { CA }\end{array}$ & $\begin{array}{l}\text { Habitación } \\
\text { planta }\end{array}$ & $\begin{array}{l}\text {-Información estudio } \\
\text {-Firma } \\
\text { consentimiento } \\
\text {-Test STAI A/E + A/R } \\
\text { ingreso } \\
\text {-Datos socio- } \\
\text { demográficos }\end{array}$ \\
\hline $\begin{array}{l}\mathbf{2}^{\circ} \text { día } \\
\text { Intervención } \\
\text { quirúrgica } \\
\end{array}$ & \begin{tabular}{|l} 
Sala \\
preanestesia \\
Alta \\
reanimación \\
\end{tabular} & $\begin{array}{l}\text {-Test STAI A/E pre } \\
\text {-Dolor EVA alta } \\
\text { rea }\end{array}$ & \begin{tabular}{|l|}
$2^{\circ}$ día \\
Intervención \\
quirúrgica \\
\end{tabular} & $\begin{array}{l}\text { Sala } \\
\text { preanestesia } \\
\text { Alta reanimación }\end{array}$ & $\begin{array}{l}\text {-Test STAI A/E pre } \\
\text {-Dolor EVA alta rea }\end{array}$ \\
\hline $3^{\text {er }}$ día & $\begin{array}{l}\text { Consulta } \\
\text { curso clínico }\end{array}$ & $\begin{array}{l}\text {-Dolor EVA post } \\
24 \mathrm{~h} .\end{array}$ & $3^{\text {er }}$ día & $\begin{array}{l}\text { Consulta curso } \\
\text { clínico }\end{array}$ & -Dolor EVA post $24 \mathrm{~h}$. \\
\hline $4^{\circ}$ día & $\begin{array}{l}\text { Consulta } \\
\text { curso clínico }\end{array}$ & $\begin{array}{l}\text {-Dolor EVA post } \\
48 \mathrm{~h} \text {. }\end{array}$ & $4^{\circ}$ día & $\begin{array}{l}\text { Consulta curso } \\
\text { clínico }\end{array}$ & -Dolor EVA post $48 \mathrm{~h}$. \\
\hline $5^{\circ}$ día & $\begin{array}{l}\text { Habitación } \\
\text { planta }\end{array}$ & $\begin{array}{lll}\text {-Test } & \text { STAl } & \mathrm{A} / \mathrm{E} \\
\text { post 72h } & \\
\text {-Dolor EVA } & \text { post } \\
72 \mathrm{~h} & & \\
\end{array}$ & $5^{\circ}$ día & $\begin{array}{l}\text { Habitación } \\
\text { planta }\end{array}$ & $\begin{array}{l}\text {-Test STAI A/E post } \\
72 \mathrm{~h} \\
\text {-Dolor EVA post } 72 \mathrm{~h}\end{array}$ \\
\hline
\end{tabular}

Para el análisis de los datos se utilizó el paquete estadístico SPSS 17.0 para Windows. Las variables categóricas (datos sociodemográficos) se describieron con frecuencias y porcentajes, mientras que las variables cuantitativas (ansiedad, dolor, bienestar, información) se resumieron con la media y la desviación estándar.

Posteriormente se realizó la prueba de normalidad con el test de Kolmogorov-Smirnov para comprobar la distribución de las variables cuantitativas. Como no se obtuvo una distribución normal en la totalidad de las variables, se decidió continuar el análisis bivariante con las pruebas no paramétricas.

Para comprobar si existía relación entre variables categóricas respecto al grupo (control e intervención), se utilizó el test Chi-Cuadrado de Pearson. Para las variables cuantitativas (centiles de nivel de ansiedad-estado y dolor escala EVA) y las variables categóricas ordinales (bienestar) se utilizó el test $U$ de Mann-Whitney para muestras independientes. Posteriormente se compararon las medias de los niveles de ansiedad-estado con la experiencia vivida (positiva o negativamente) en procesos quirúrgicos anteriores y la información recibida (suficiente o insuficiente).

Para comprobar si existía relación entre el nivel de ansiedad-estado y el dolor, se utilizó la correlación Rho de Spearman.

Se calcularon intervalos de confianza al $95 \%$ de seguridad, con un nivel de significación de $p<0,05$. 


\section{RESULTADOS}

La edad media de los pacientes del grupo control fue de $67^{\prime} 87$ años $\left(D E=12^{\prime} 7\right)$ con un rango de 33 a 83 años, mientras que en el grupo intervención, la edad media fue de $67^{\prime} 40$ años (DE=11'73) con un rango de 42 a 81 años.

El tipo de intervenciones realizadas fue similar en ambos grupos. En el grupo control: artrodesis lumbar $(20 \%)$, prótesis total de rodilla $(56,7 \%)$ y prótesis total de cadera $(23,3 \%)$. En el grupo intervención: artrodesis lumbar $(26,7 \%)$, prótesis total de rodilla $(50 \%)$ y prótesis total de cadera $(23,3 \%)$. Hay que destacar la mayor participación de mujeres $(80 \%)$ en el grupo control respecto al grupo intervención $(56,7 \%)$.

Para analizar la evolución de los niveles de ansiedad-estado en todo el proceso se calculó la diferencia entre distintos momentos, resultando el más destacado la diferencia entre el momento del ingreso, el día anterior a la operación, y la sala de preanestesia, justo antes de entrar en quirófano. Tal como se puede observar en el gráfico 1, el grupo control experimentó un aumento del nivel de ansiedad (15,83 puntos), mientras que en el grupo intervención, éste disminuyó (3,03 puntos), siendo la diferencia de la evolución estadísticamente significativa $(p=0,001)$.

Gráfico 1. Evolución de los niveles de ansiedad en diferentes momentos evaluados (NC 95\%).

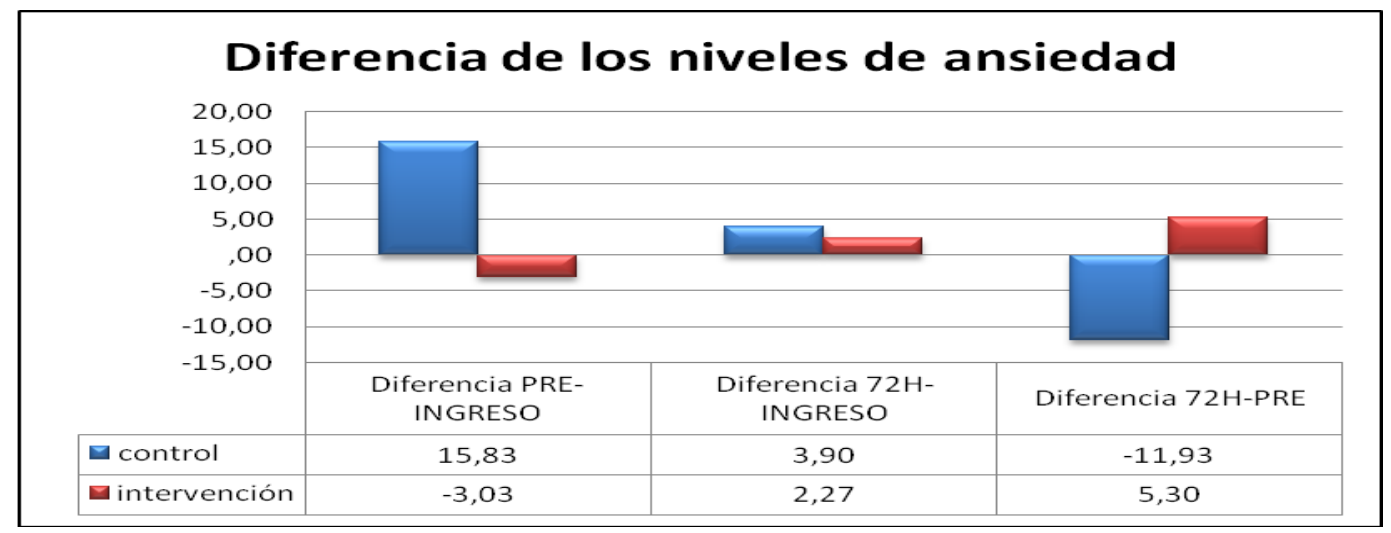

El gráfico 2 muestra la evolución de la percepción del dolor según la Escala Visual Analógica (EVA), en el que se observa que el grupo control tenía una EVA superior al grupo intervención en los cuatro momentos del proceso. Esta diferencia fue estadísticamente significativa a las 24,48 y 72 horas postoperatorias con un $p$-valor asociado inferior a 0'05 ( $p=0,043,0$,001 y 0'017 respectivamente). 
Gráfico 2. Evolución de la percepción del dolor en los diferentes momentos evaluados.

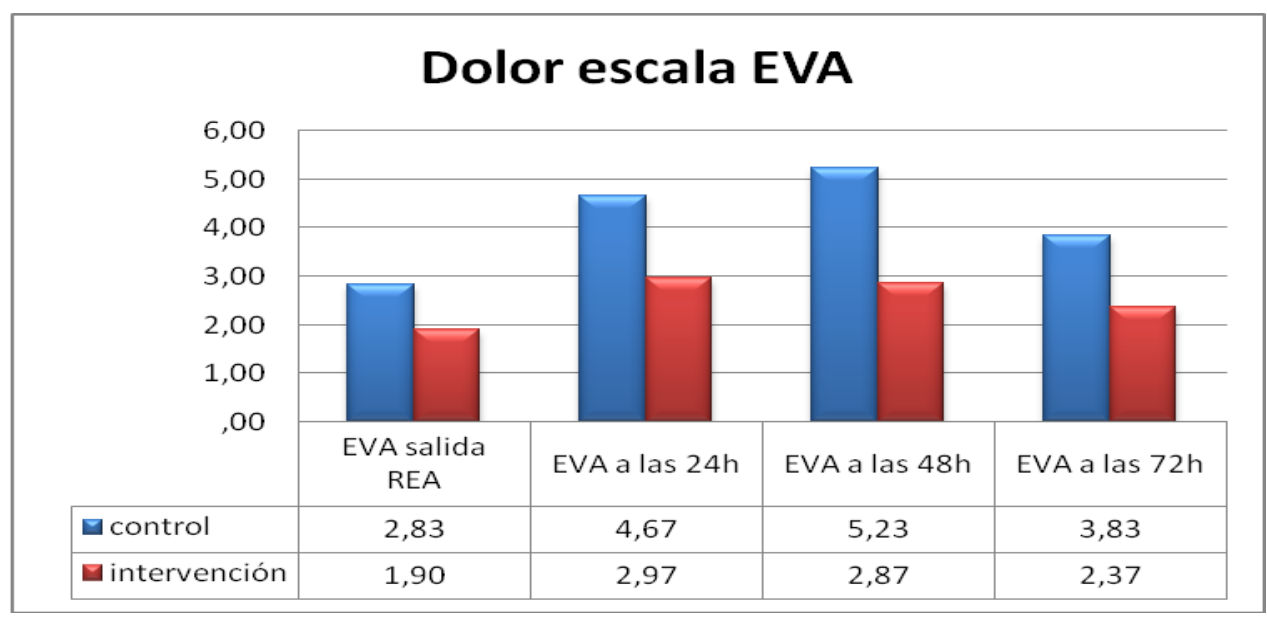

Posteriormente se comparan los niveles de ansiedad-estado en relación a si las experiencias quirúrgicas anteriores se habían vivido de manera positiva o negativa. El gráfico 3 constata que los pacientes que habían tenido una experiencia previa positiva tenían unos niveles de ansiedad inferiores a los pacientes con experiencias previas negativas. Esta diferencia fue estadísticamente significativa con $p$-valores inferiores a 0 05 ( $p=0,046,0,017$ y 0,005 respectivamente).

Gráfico 3. Nivel de ansiedad por experiencia previa

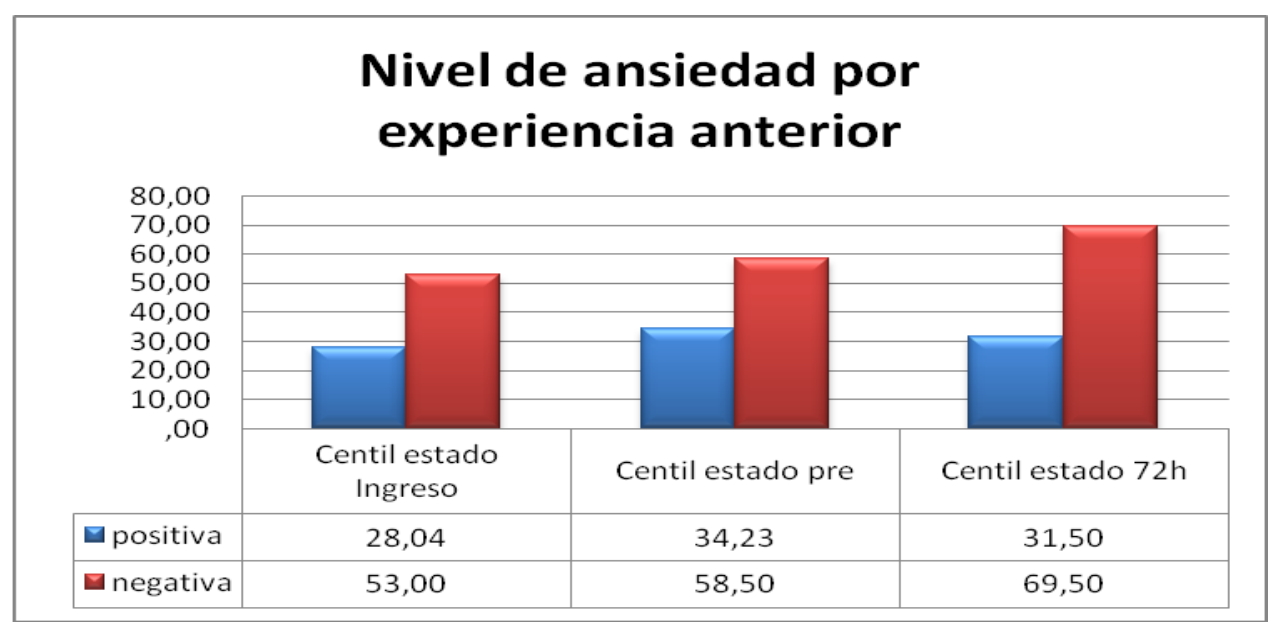

Respecto a la información recibida, el grupo que la consideró suficiente presentó un nivel de ansiedad-estado estadísticamente inferior al grupo que la consideró insuficiente $(p=0,039)$ en el momento del ingreso.

En relación a la evolución de la sensación de bienestar se observaron diferencias estadísticamente significativas: el grupo intervención presentó más puntuaciones de "bastante" y "mucho" en la sala de preanestesia $(p<0,001)$ y a las 72 horas postoperatorias $(p=0,001)$ respecto al grupo control. Por el contrario, las únicas puntuaciones de "nada" correspondieron al grupo control en las mismas situaciones.

Respecto a la posible relación entre los niveles de A/E y la percepción del dolor, los coeficientes de Rho de Spearman oscilaron entre 0'310 y 0'661, datos que indican la existencia de una asociación positiva moderada a sustancial, en concreto entre los 
niveles de $A / E$ en el momento del ingreso y la sala de preanestesia $(p=0,661)$ y a las 72 horas postoperatorias $(p=0,599)$. También hay que destacar la relación positiva moderada entre los niveles de $A / E$ en la preanestesia y el dolor postoperatorio a las 24 horas $(p=0,344)$, a las 48 horas $(p=0,499)$ y a las 72 horas $(p=0,463)$.

\section{DISCUSIÓN Y CONCLUSIÓN}

Este trabajo evidencia que las personas que presentan un nivel de $\mathrm{A} / \mathrm{E}$ elevado en el momento del ingreso el día anterior a la intervención quirúrgica, experimentan un aumento significativo del nivel de A/E en la sala de preanestesia, y, en consecuencia, tienen una percepción del dolor significativamente superior a las 24, 48 y 72 horas postoperatorias.

Estos resultados están en la misma línea que otros estudios que evalúan la importancia de la información previa a una intervención quirúrgica ${ }^{31,37,42}$ y la eficacia de la visita prequirúrgica estructurada de enfermería para disminuir tanto la ansiedad en el preoperatorio inmediato como la percepción del dolor postoperatorio durante las primeras 72 horas $^{1,4,7}$.

Similar a los estudios de Heluy et al. ${ }^{30}$ y Balaguer $^{5}$, se ha considerado a la familia como un elemento integrado en el proceso y se ha motivado a sus miembros para que estuviesen presentes durante la visita prequirúrgica y expresaran también sus dudas. En algunos casos, la actitud de los familiares fue determinante, ya que algunas personas de edad avanzada y sin estudios mostraban dudas en la participación del proyecto, y la disposición positiva de la familia contribuyó y facilitó la recogida de datos.

Respecto a la mejora de la sensación de bienestar descrita en la teoría del Caring de Jean Watson, ésta mejoró significativamente en las personas que habían recibido la información durante la visita prequirúrgica, en concreto en la sala de preanestesia y a las 72 horas postoperatorias, coincidiendo con los estudios publicados, tanto en nuestro entorno cultural como en el contexto anglosajón 1,4,7,10,37,38,43,48-50.

A diferencia de lo que afirman Kindler et al. ${ }^{47}$ y Herrera-Espiñeira et al. ${ }^{46}$, el nivel de estudios no influyó de manera estadísticamente significativa en el nivel de ansiedad ni en la percepción del dolor postoperatorio, a pesar de que los pacientes sin estudios presentaron puntuaciones más elevadas.

Debido a la mejora de las técnicas quirúrgicas y anestésicas, se ha conseguido reducir los días de hospitalización de las personas intervenidas quirúrgicamente, y en los últimos años ha aumentado el tipo de cirugía con complejidad que se practica en régimen de CMA (Cirugía Mayor Ambulatoria) y CMI (Cirugía Mínimamente Invasiva) y que ingresan el mismo día de la intervención ${ }^{12,52}$. Paradójicamente, con estos avances técnicos, se pierde la posibilidad de realizar la visita prequirúrgica el día anterior a la intervención, circunstancia que implica una disminución del contacto entre enfermera-paciente antes de la cirugía, y por lo tanto, se pierde la posibilidad de dar la información necesaria con el objetivo de disminuir la ansiedad.

Como conclusión de este trabajo, los resultados evidencian que la visita prequirúrgica estructurada de enfermería es una herramienta eficaz para disminuir la ansiedad en los momentos previos a la intervención y la percepción del dolor postoperatorio durante las primeras 72 horas, así como mejora la percepción de bienestar.

Para avanzar en los cuidados de enfermería y humanizar nuestra asistencia hay que remodelar los sistemas organizativos que se han quedado arcaicos para cubrir las necesidades de nuestra población ${ }^{53}$. Hay que luchar para crear consultas de 
enfermería paralelas a las consultas de los anestesistas con el objetivo de que todos los pacientes puedan beneficiarse de los efectos ansiolíticos de la actuación de la enfermera en el proceso preoperatorio desde una perspectiva holística, incluidos los que son intervenidos en régimen de CMA y $\mathrm{CMI}$, con lo que se podría disminuir la ansiedad en la semana anterior al ingreso hospitalario, tal como defienden numerosos artículos publicados $2,6,19,33,42,43$.

Proponemos nuevas líneas de investigación con metodología cualitativa para identificar vivencias personales y necesidades relacionadas con la asimilación de la información con el objetivo de publicar y divulgar los resultados para contribuir en el avance de la disciplina enfermera y mejorar la calidad asistencial en todos los niveles.

\section{REFERENCIAS}

1.- Orihuela Pérez I, Aranda Salcedo T, Escobar Julián F, González Ramírez A, Jiménez Ruiz R, Martínez García A, et al. Visita preoperatoria de enfermería: evaluación de la efectividad de la intervención enfermera y percepción del paciente. Enfermería Clínica. 2010;20(6):349-354.

2.- Carapia-Sadurni A, Mejía-Terrazas GE, Nacif-Gobera L, Hernández-Ordóñez MN. Efecto de la intervención psicológica sobre la ansiedad preoperatoria. Revista Mexicana de Anestesiología. 2011;34(4):260-263.

3.- de la Paz-Estrada C, Prego-Beltrán C, Barzaga-Hernández E. Miedo y ansiedad a la anestesia en pacientes sometidos a cirugía. Revista Mexicana de Anestesiología. 2006;29:159-162.

4.- Aparcero Bernet L, Núñez García A, Ramos Fernández M, Zahino Ruiz G, Olcina Santonja R. La visita entrevista prequirúrgica: Intervención de enfermera e instrumento de calidad. Enfuro. 2003(88):9-13.

5.- Balaguer de Dios M. Protocolo de acogida e información preoperatoria para pacientes quirúrgicos. Revista de la Asociación Española de Enfermería Quirúrgica. 2006(22):7-13.

6.- Valenzuela-Millán J, Barrera-Serrano JR, Ornelas-Aguirre JM. Ansiedad preoperatoria en procedimientos anestésicos. Cirugía y Cirujanos. 2010;78(2):151-156 7.- Chirveches E, Arnau A, Soley M, Clotet G, Roura P, et al. Efecto de una visita prequirúrgica de enfermería perioperatoria sobre la ansiedad y el dolor. Enfermería Clínica. 2006;16(1):3-10.

8.- Martín-Crespo Blanco MC, Caravantes Marín MI, García Mas JM, Gómez González B. Efecto de la intervención enfermera durante el intraoperatorio en el grado de ansiedad del paciente quirúrgico en el hospital comarcal Virgen de Altagracia de Manzanares (Ciudad Real). Nure Investigation. 30.

9.- Moix Queraltó J. Disminución de la ansiedad como factor de mejora de la calidad asistencial en pacientes quirúrgicos. Revista de Calidad Asistencial. 1998;13:160-165. 10.- Moix Queraltó J. Emoción y cirugía. Anales de Psicología. 1994;10(2):167-175.

11.- Munafò MR, Stevenson J. Anxiety and surgical recovery: Reinterpreting the literature. Journal of Psychosomatic Research. 2001;51(4):589-596.

12.- Mclntosh S, Adams J. Anxiety and quality of recovery in day surgery: A questionnaire study using Hospital Anxiety and Depression Scale and Quality of Recovery Score. International Journal of Nursing Practice. 2011;17(1):85-92.

13.- Caumo W, Schmidt AP, Schneider CN, Bergmann J, Iwamoto CW, Bandeira D, et al. Risk factors for preoperative anxiety in adults. Acta Anaesthesiologica Scandinavica. 2001;45(3):298.

14.- Vaughn F, Wichowski H, Bosworth G. Does preoperative anxiety level predict postoperative pain? AORN Journal. 2007;85(3):589-604. 
15.- Kagan I, Bar Tal Y. The effect of preoperative uncertainty and anxiety on shortterm recovery after elective arthroplasty. Journal of Clinical Nursing. 2008;17(5):57683.

16.- Varela Curto MD, Villamisar Ferreiro MJ, Sanjurjo Gómez ML, Lamata Martínez A. Plan estandarizado de cuidados intraoperatorios. Enfuro. 2004(90):25-29.

17.- Mitchell M. Conscious surgery: Influence of the environment on patient anxiety. Journal of advanced Nursing. 2008;64(3):261-271.

18.- Mitchell M. Patient anxiety and conscious surgery. Journal of Perioperative Practice. 2009;19(6):168-173.

19.- Mitchell M. Patient anxiety and modern elective surgery: a literature review. Journal of Clinical Nursing. 2003;12(6):806.

20.- Marín Romero I, Martínez Gómez P, Ponsich Pubill J, Pubill Grasa M. Ansiedad en paciente intervenidos de rodilla (Prótesis Total Rodilla). Enfermería Global. 2004:4:1-11.

21.- Eriksson K. Caring science in a new key. Nursing Science Quarterly. 2002;15(1):61-65.

22.- Fawcett J, Watson J, Neuman B, Walker PH, Fitzpatrick JJ. On nursing theories and evidence. Journal of Nursing Scholarship. 2001;33(2):115-119.

23.- Gordillo León F, Arana Martínez JM, Mestas Hernández L. Tratamiento de la ansiedad en pacientes prequirúrgicos. Revista Clínica de Medicina de Familia. 2011;4(3):228-233.

24.- Watson J. Caring theory as an ethical guide to administrative and clinical practices. Nursing Administration Quarterly. 2006;30(1):48.

25.- Cara C. A pragmatic view of Jean Watson's caring theory. International Journal for Human Caring. 2003;7(3):51-61.

26.- Watson J. Caring science and human caring theory: Transforming personal and professional practices of nursing and health care. Journal of Health and Human Services Administration. 2009;31(4):466-482.

27.- Norred CL. Minimizing preoperative anxiety with alternative caring-healing therapies. AORN. 2000;72(5):838-843.

28.- Marriner Tomey A, Raile Alligood M. Filosofía y ciencia del cuidado. Modelos y teorías en enfermería. Quinta ed. Barcelona: Elsevier; 2007. p. 91-107.

29.- Rudolfsson G, von Post I, Eriksson K. The expression of caring within the perioperative dialogue: A hermeneutic study. International Journal of Nursing Studies. 2007;44(6):905-915.

30.- Heluy de Castro C, Efigênia de Faria T, Felipe Cabañero R, Castelló Cabo M. Humanización de la Atención de Enfermería en el Quirófano. Index de Enfermería. 2004;13(44-45):18-20.

31.-Egbert LD, Battit G, Turndorf H, Beecher HK. The value of the preoperative visit by an anesthetist. A study of doctor-patient rapport. JAMA. 1963;185(7):553.

32.- Blanca Gutiérrez JJ. La educación pre-operatoria: análisis de contenidos y de su influencia en la recuperación del paciente. Inquietudes: Revista de Enfermería. 2005;11(31):34-36.

33.- Díez-Álvarez E, Arrospide A, Mar J, Alvarez U, Belaustegi A, Lizaur B, et al. Efectividad de una intervención preoperatoria de enfermería sobre el control de la ansiedad de los pacientes quirúrgicos. Enfermería Clínica. 2011. doi:10.1016/j.enfcli.2011.09.005

34.- Hughes S. The effects of giving patients pre-operative information. Nursing Standard (Royal College of Nursing). 2002;16(28):33.

35.- Kiyohara LY, Kayano LK, Oliveira LM, Yamamoto MU, Inagaki MM, Ogawa NY, et al. Surgery information reduces anxiety in the pre-operative period. Rev Hosp Clin Fac Med S Paulo. 2004;59(2):51-56. 
36.- Maward L, Azar N. Comparative study of anxiety between informed and notinformed patients in preoperative period. Recherche en Soins Infirmiers. 2004(78):3558.

37.- McDonald S, Hetrick S, Green S. Educación prequirúrgica para el reemplazo de cadera o de rodilla. La Biblioteca Cochrane Plus. 2008;núm.2.

38.- Gallego J, Rodríguez de la Torre, MR, Vázquez-Guerrero J, Gil M. Estimación de la prevalencia e intensidad del dolor postoperatorio y su relación con la satisfacción de los pacientes. Revista de la Sociedad Española del Dolor. 2004;11(4):35-40.

39.- Rodríguez Navarro JC, Gómez Gracia E, Fernández-Crehuet Navajas J. El impacto emocional de la información médica en pacientes quirúrgicos. Cuadernos de Bioética. 2001(12):367-378.

40.- Sjoling M, Nordahl G, Olofsson N, Asplund K. The impact of preoperative information of state anxiety, postoperative pain and satisfaction with pain management. Patient Education and Counseling. 2003;51(2):169-76.

41.- Spalding NJ. Reducing anxiety by pre-operative education: Make the future familiar. Occupational Therapy International. 2003;10(4):278-293.

42.- Stergiopoulou A, Vlachos $\mathrm{G}$. The preoperative education effect on the reduction of patient anxiety. Noseleutike. 2010;49(1):26-30.

43.- Walker J. Patient information. What is the effect of preoperative information on patient satisfaction? British Journal of Nursing. 2007;16(1):27-32.

44.- Navas Monzón M. Nivel de ansiedad del paciente quirúrgico en el preoperatorio y postoperatorio inmediato y factores de riesgo. Cuidando la salud. 2008(8):36-51.

45.- Adeline CYM. Patients Perspectives on the Pre-Operative Education Programme. 2003.SGH Proceedings. 2003;12(2):64-71.

46.- Herrera-Espiñeira C, Quero-Rufián A, Martínez-Cirre C, Rodríguez del Castillo M, Rodríguez del Águila, MM, Aguayo de Hoyos E. Información recibida por los pacientes acerca de su estado de salud y su comprensión, antes y durante su hospitalización. Enfermería Clínica. 2006;16(4):184-189.

47.- Kindler $\mathrm{CH}$, Harms C, Amsler F, Ihde-Scholl T, Scheidegger D. The visual analog scale allows effective measurement of preoperative anxiety and detection of patients' anesthetic concerns. Anesthesia \& Analgesia. 2000;90(3):706-712.

48.- Williams O. Patient knowledge of operative care. Journal of the Royal Society of Medecin. 1993;86(6):328.

49.- Gallego J, Rodríguez de la Torre, MR, Vázquez-Guerrero J, Gil M. Estimación de la prevalencia e intensidad del dolor postoperatorio y su relación con la satisfacción de los pacientes. Revista de la Sociedad Española del Dolor. 2004;11(4):35-40.

50.- González-Valentín MA, Padín S, De Ramón E. Satisfacción del paciente con la atención de enfermería. Enfermería Clínica. 2005;15(3):147-155.

51.- Spielberger CD, Gorsuch RL, Lushene RE. STAI: cuestionario de ansiedad estado-rasgo. Cuarta ed. Madrid: TEA; 1994.

52.- Gilmartin J, Wright K. The nurse's role in day surgery: a literature review. International Nursing Review. 2007;54(2):183-190.

53.- Jiménez-Castellanos MH. La visita prequirúrgica como intervención enfermera: ¿humanización o automatización de los cuidados?. Evidentia. 2007 nov-dic; 4(18). En: http://www.index-

f.com/evidentia/n18/396articulo.php [ISSN:1697638X]. Consultado el 31 de Enero de 2013. 
Recibido: 3 de marzo 2014; Aceptado: 31 de marzo 2014 